\title{
Building Robota, a Mini-Humanoid Robot for the Rehabilitation of Children with Autism Aude Billard $^{1}$, Ben Robins ${ }^{2}$, Jacqueline Nadel $^{3}$, Kerstin Dautenhahn ${ }^{2}$ \\ 1: LASA Laboratory, School of Engineering, EPFL, Lausanne CH 1015, Switzerland \\ 2: Adaptive Systems Research Group, University of Hertsfordshire, U.K. 3: CNRS-UMR 7593, Hopital de la Salpetriere, Paris, France \\ Corresponding Author: aude.billard@epfl.ch, \\ http://lasa.epfl.ch
}

\begin{abstract}
The Robota project constructs a series of multiple degrees of freedom doll-shaped humanoid robots, whose physical features resemble those of a human baby. The Robota robots have been applied as assistive technologies in behavioral studies with low-functioning children with autism. These studies investigate the potential of using an imitator robot to assess children's imitation ability and to teach children simple coordinated behaviors. In this paper, we review the recent technological developments that have made the Robota robots suitable for use with children with autism. We critically appraise the main outcomes of two sets of behavioral studies conducted with Robota and discuss how these results inform future development of the Robota robots and generally, robots for the rehabilitation of children with complex developmental disabilities.
\end{abstract}

\section{Introduction:}

Robota is the name of a series of doll-shaped mini-humanoid robots. The Robota project is part of a current trend of robotics research that develops educational robot toys; see, e.g. [Michaud \& Caron 2002, Plaisant et al 2000, Kozima et al 2002]. Such a trend aims at understanding the role that an "entertaining" robot might play in the educational development of the child. This approach goes beyond building playful robots, whose sole purpose is that of entertaining the user, e.g. [Fujita et al 2003, Sawada et al 2004], by stressing the educational and therapeutic role of the robot [Dautenhahn 1999].

The use of robots as entertainment systems puts important constraints on the design of both the physical appearance of the robot and of its control system. For instance, the physical appearance of an entertaining robot should match a number of aesthetic characteristics to make it look playful and engaging [Breazeal 2002, Kitano \& Fujita 2000; Montemayor et al, 2000]. In contrast, designing a robot for use with children with disabilities may, in some cases, put different constraints on the robot's physical appearance. First, the robot will have to be robust beyond classical standards [Michaud \& Theberge-Turmel, 2002] and it may not necessarily be advantageous to display human features. For instance, [Robins et al, 2004] showed that children with autism would interact more when the robot's face is plain (no facial features) than when it bears a strong resemblance to that of a human. 
In [Michaud \& Caron 2002, Dautenhahn 1999, Dautenhahn \& Werry 2002], the robot bears no similarity to the human body, and the interaction is based on coordinated motion of the child and robot in space. The focus is, in this case, on the interactive and coordinated aspects of the robot's behaviour. In contrast, in [Plaisant et al 2000, Kozima et al 2002, 2005, Scasselatti 2005] and in the Aurora project [Robins et al 2004a/b, Dautenhahn \& Billard 2002], the robot shares, at least in part, a similar embodiment to that of the child. In [Kozima, 2002], the robot is provided with a complete humanoid torso including arm and head. This high degree of human likeliness is exploited to simulate a number of fundamental social interactions, such as pointing, joint attention, and gaze directionality in reaching. Other works have shown, however, that such human-like interaction may be elicited with much simpler designs. For instance, in [Plaisant et al 2000], the robot consists of a fluffy animal with two motorized arms. The robot can engage in mirror imitative behaviour by the flapping of its two arms. While the general features of the robot do not resemble those of a human, the body symmetries created by the head and the two arms are sufficient to elicit in the children an understanding of the simple imitative game. In [Kozima et al 2005], the robot is a small "snowman-like" puppet with no arms but an expressive face that conveys a feeling of purpose and intent when looking at the kid. Such a simple design was sufficient to elicit spontaneous dyadic interactions. Finally, in [Scasselatti 2005] a simple anthropomorphic face, remotely controlled and displaying simple emotions, was shown to engage children with autism in interactive and playful sessions.

The Robota project follows such work by emphasizing the humanoid aspect of the robot, and, especially the human-like appearance of the robot's face. Studies with Robota have two goals: 1) to test systematically the reaction of low-functioning children with autism to the different human features of the robot; 2) to evaluate the extent to which low-functioning children with autism are able to distinguish between perceptions being the result of their own actions and perceptions being the result of actions of others. To measure the latter, Robota engages the children in simple imitation games, using the legs, arms and head.

In this paper, we review the recent technological developments that have made the Robota robots suitable for use with children with autism. Section 2 gives an overview of the technological developments behind the current Robota robot. In Section 3, we critically appraise the main outcomes of two sets of behavioural studies conducted with Robota and in Section 4, we discuss how these results inform future development of the Robota robots and generally, robots for the rehabilitation of children with complex developmental disabilities.

\section{Robota: Technological Developments}

\subsection{Design considerations}

The use of Robota as part of experiments with children with autism sets a number of constraints on the design of the robot's mechanics and electronics. These are: 


\section{Ease of Set-up:}

- The robot's size and weight must be sufficiently small, so that it could be easily moved around in the room by the experimenter ${ }^{1}$ and, if necessary, be carried about by a young child ${ }^{2}$. We take, as reference, the average size and weight of commercial dolls, namely $50 \mathrm{~cm}$ in height and $1 \mathrm{~kg}$ in weight.

- The processing must be done on-board and the robot should be battery-operated for easy setup in the experimental room.

- The cost of the robot must be sufficiently low for the collaborating schools and museums to eventually be able to purchase it.

\section{Appearance and Behaviour:}

- The robot's body and facial features must resemble those of a human. Robota provides a bridge between non-human looking machines with which children with autism can engage in social and playful behaviours and human beings toward which these kids are known to be less engaged in interacting socially. In Section 3.1, we briefly summarize experiments that contrast the children's reaction to the human appearance of Robota's face to a non-human face.

- The robot must be "child-friendly". This motivated our use of a commercially available doll. We wished to present the kids with an object they would be accustomed to playing with at schools and at home. The assumption was that if the robot was to look exactly like a commercial doll the children are used to play with or at least are exposed to in their everyday life, then, we could rule out the surprise effect that this robot may have on them. After discussing with the therapists and educators in the school we visited, it was readily accepted that a doll, such as Robota, would be part of the general repertoire of toys the kids would have been accustomed to at school. It has, indeed, been the case that so far the robot has been well accepted by all children. Moreover, in one instance, during the test conducted at the Hospital de la Salpetriere, one child realized that Robota was somewhat similar to a doll he used to play with at school and went to fetch the doll to compare it physically to Robota, see Section 3.2 .

- While originally, we provided the robot with multiple capabilities to allow multimodal interaction, it quickly became clear that, in order to pursue systematic studies, one should use only a subset of these capacities. The current experiments with Robota aimed at measuring the children with autism's ability to respond to a simple interactive imitation game involving the whole body. Because of economical constraints on the total number of DOFs, we chose to assign no more than 5 degrees of freedom (DOF) to drive each of the robot's limbs separately.

\footnotetext{
${ }^{1}$ The experimenters must adapt the positioning of the robot to the rooms they are assigned to in the schools, in order not to disrupt the normal distribution of objects, which the children are used to.

${ }^{2}$ The later constraint does not apply to the experiments reported here. However, in the long term, our goal is to provide Robota as a companion/educational robot for home use.
} 


\subsection{Robota Basic Hardware}

The Robota project started in 1997 with the first prototype created out of LEGO parts and plastic components of a commercial doll. See Figure 1. The original robot was equipped with a pair of infrared (IR) detectors to track the motions of the user's arms and head. Each infra-red detector consisted of an emitter and a receptor. Two of the IR receptors were placed on the robot's chest and measured the signals of the corresponding IR emitters, which the user held, one in each hand. The user sat or stood facing the robot. The signal received by each sensor on the robot's chest was used to control each of the robot's arms. That is, when the user moved his/her left arm in front of the robot, the right detector on the robot was activated, in turn triggering movement in the robot's right arm. These waving movements consisted of lifting the arm up and then down at a fixed frequency. The two other IR emitters were placed on the robot's ears, while the two corresponding IR receptors were mounted onto a pair of glasses, which the user wore. Phototaxis ${ }^{3}$ was performed on the two IR signals and used to direct the robot's head. That is, when the user looked, e.g. to the left, the left detector on the glasses received full activation while the right one received none. This triggered the robot's head movement to the left, i.e. the robot turned the head to the left (and vice-versa for the right). After a fixed time delay (about half a second), the robot moved its head back to its neutral position, facing the user. As a result, the robot appeared to mirror the user's arm and head movements.

This first prototype was used in a first batch of tests with seven children with autism as part of the Aurora project [Dautenhahn \& Billard 2002], see also Section 3. While the children quickly understood the game, it was also quite clear that wearing the glasses was cumbersome, and, ill-suited for children with autism, who usually dislike wearing foreign accessories. Moreover, it also appeared important to make the robot more robust in order to allow children to touch it without risk of breaking it.

\footnotetext{
${ }^{3}$ Phototaxis is the movement of an organism or agent toward or away from a source of light.
} 

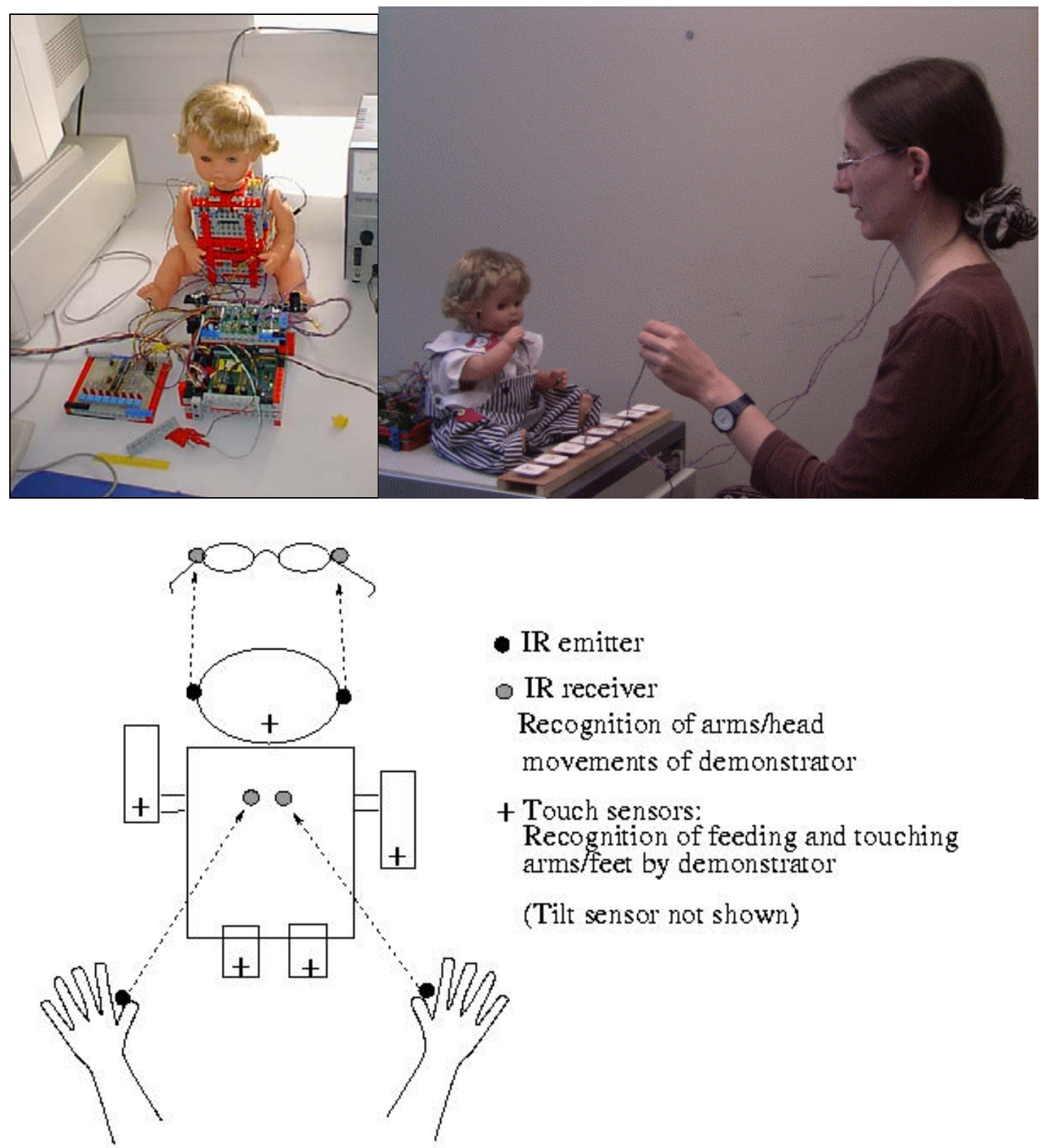

IR emitter

- IR receiver

Recognition of armshead

movements of demonstrator

+ Touch sensors:

Recognition of feeding and touching arms/feet by demonstrator

(Tilt sensor not shown)

Figure 1: (Top) First prototype of Robota, made of LEGO parts. (Bottom) Schematic of the pair of infra-red detectors (emitter-receiver) driving the imitation game in the first prototype.

Thus, numerous iterations thereafter followed, leading to the creation of a commercial prototype of Robota, sold by DIDEL SA, see Figure 2. 


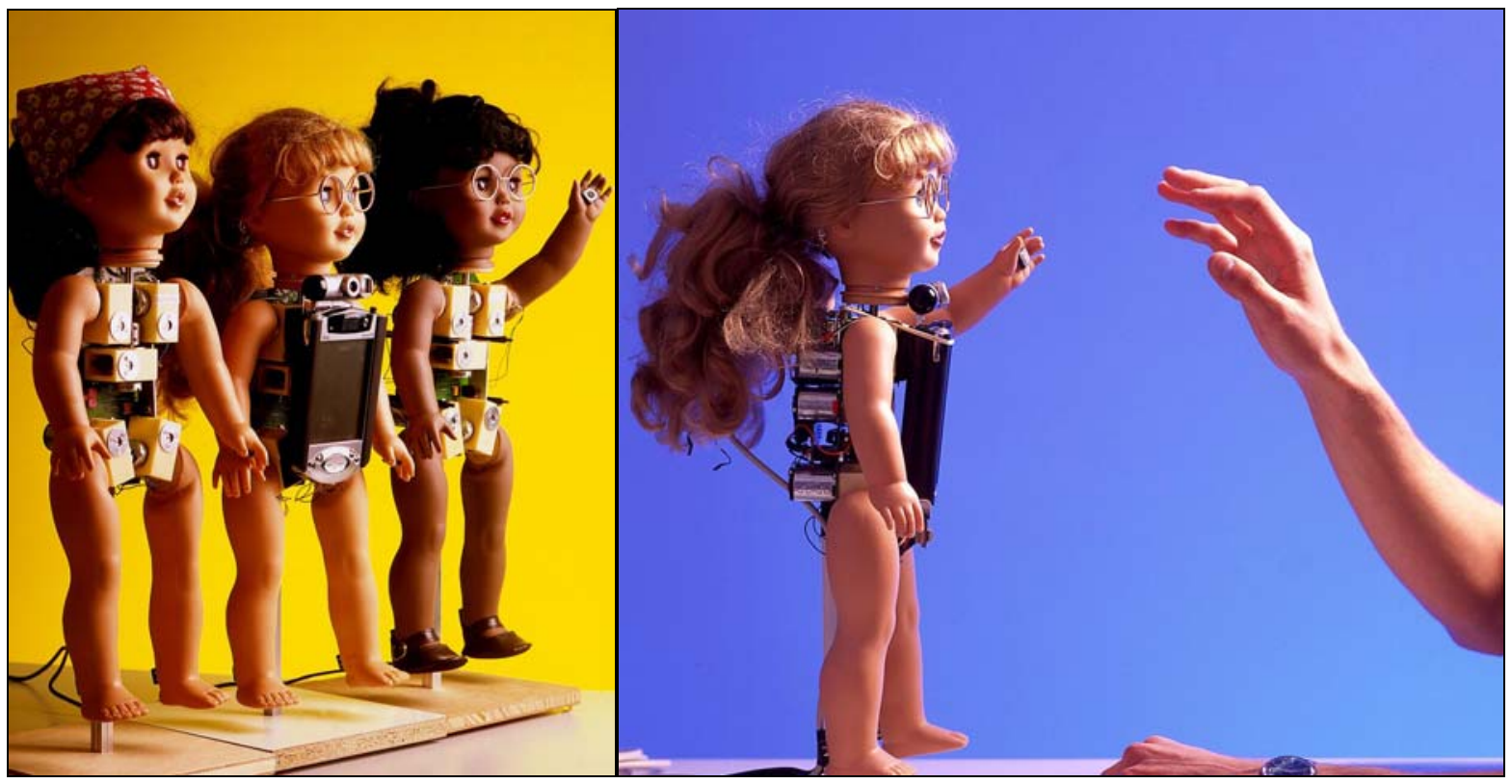

Figure 2: LEFT: 3 Robota robots. The middle one is provided with a Compaq iPAQ 3850 as main CPU. RIGHT: A camera mounted on the PocketPC of Robota enables tracking of the hands and nose of the user through optical flow and recognition of skin colour. Robota can, then, mirror the movements of the arm and head of the user.

The Robota robot, sold currently by DIDEL SA ${ }^{4}$ at a price of 3000.- (US\$), has 5 degrees of freedom (DOF): 1 DOF for each leg, arm and head. DC motors with a 1:6 gear ratio drive the 5 DOFs, providing a maximum continuous torque of about $90 \mathrm{mNm}$. Robota's electronic components consist of a Motor Board and a Sensor Board. The Motor board is addressed via a RS232 serial interface from a PC or a PocketPC. The Sensor Board is addressed via a SPI (serial peripheral interface) serial interface. An external keyboard can also be attached through an extra SPI port on the sensor board. The Motor Board acts as the master of the SPI connection with Sensor Board and external keyboard. The Motor Board, Sensor Board and external keyboard are controlled by a PIC (programmable Interupt Controller) microcontroller.

In the experiments reported in Section 3, the Robota CPU is provided by a Compaq iPAQ-3850 palm top computer. The PocketPC is mounted on the front of the robot, see Figure 2 and communicates with the robot via a RS232 serial interface. The iPAQ is equipped with a FlyCAM-CF camera. The camera faces the user, taking snapshots with a resolution of $160 \times 120$ pixels at a rate of 15 frames per second. The iPAQ processor is a StrongARM 32-bit RISC Processor working at $206 \mathrm{MHz}$, with $64 \mathrm{Mb}$ of RAM. The FlyCam-CF camera is connected via a CompactFlash Memory Card, through the PocketPC Expansion Pack. More technical specifications are given in [Calinon \& Billard, 03].

Table 2 lists the accessories used in the different applications of Robota, see Section 3.

\footnotetext{
${ }^{4}$ www.didel.com
} 
The camera mounted on the PocketPC of Robota enables tracking of the hands and nose of the user through optical flow and recognition of skin colour, see [Calinon \& Billard, 03]. Robota can, then, mirror the movements of the arm and head of the user. The camera set-up replaces the infra-red set-up of the first prototype (see Figure 1) providing a means of tracking the children's motion, that is more acceptable to children with autism. Indeed, this way the user does not have to wear any accessories.

Robota is also provided with a set of switches, placed within the palms of the robot and on the ankles of the robot's feet. While each motor is endowed with potentiometers, and, thus, passive motion applied to the joint could be detected, we thought, in an early version, that having switches (more visible than the potentiometers) would entice the children to touch the robot. When touched (i.e. once a switch got hit), the robot would react by moving the corresponding limb. In a preliminary test conducted in 1998 and 1999, we experimented with such a set of switches. It quickly appeared that using the switches added too much variability to the interactions. The use of the switches was, thus, abandoned for the longitudinal studies, reported in Section 3, in order to keep the focus on the imitation game the children can play with the robot. However, these are features that remain available and will be reusable later on.

The Sensor board is equipped with a buzzer ${ }^{5}$ that can be used to play simple melodies. It is used at start-up to indicate that the robot has finished booting (similar to the windows prompt).

Table 1: Robota Technical Specifications

\begin{tabular}{|l|l|}
\hline $\begin{array}{l}\text { Height } \\
\text { Width } \\
\text { Weight }\end{array}$ & $\begin{array}{l}45 \mathrm{~cm} \\
14 \mathrm{~cm} \\
1500 \mathrm{~g} .\end{array}$ \\
\hline \hline Degrees of Freedom: & $\begin{array}{l}\text { Head rotation, raising and lowering the left/right } \\
\text { arm or left/right leg, coordinated motion of the two } \\
\text { eyes (sideway) and individual motion of each eye } \\
\text { (blinking) }\end{array}$ \\
\hline \hline Actuators & $\begin{array}{l}\text { DC Motors (Maxon A-max 26 mm) with clutches } \\
\text { and position potentiometers }\end{array}$ \\
\hline Microprocessors Motor Card & PIC - 16F870, 4MHz \\
\hline Microprocessors SensorCard & PIC - 16F84, 16MHz \\
\hline CPU & PocketPC Compaq \\
\hline Sensors & $\begin{array}{l}\text { Switches (x6), infra-red emitter/receptor (x2), } \\
\text { inclinometer (x1), buzzer (x1) }\end{array}$ \\
\hline $\begin{array}{l}\text { Battery } \\
\text { Running Time: } \\
\text { Power supply }\end{array}$ & $\begin{array}{l}7.2 \mathrm{~V}, 6 \mathrm{x} 1.2 \mathrm{NiCd} \\
\text { 30 minutes } \\
12 \mathrm{~V}, 4 \mathrm{~A} .\end{array}$ \\
\hline
\end{tabular}

${ }^{5}$ A buzzer consists of a membrane that vibrates under electrical stimulation and produces sound. 
Table 2: Robota Accessories

\begin{tabular}{|l|l|}
\hline PC Interface: & OS: Win 2000, Visual C++6.0, Serial Port (RS232 - 9600 baudrate) \\
& PocketPC-2000 \\
\hline \hline \multirow{3}{*}{ Accessories } & $\begin{array}{l}\text { QuickCam Camera or } \\
\text { FlyCAM-CF Camera } \\
\text { Speech Synthesizer (ELAN) } \\
\text { Speech Recognizer (Conversay) } \\
\text { Electronic KeyBoard (Xylophone + Joystick) } \\
\text { Drawing Pad/Touch Screen }\end{array}$ \\
\hline
\end{tabular}

\subsection{Robota's Mobile Eyes}

Recent developments have led to the creation of a 3 DOF pair of moving eyes, see [Guenter et al 2004, Pongas et al 2004] for a complete description. One DOF drives the horizontal rotation of the two eyes and the two other DOFs drive the vertical rotation of each eye (note that the eyelids are stuck on the eye, and, thus, do not move separately from the eye, see Figure 3). Thus, the robot can wink but not squint. The difficult aspect of the mechanical design was to place the center of rotation at the center of each eye, in order for the eyes to move easily within the original doll head. In each eye, we have placed one OV764X webcam (typically used in cell phones or playstation) driven by the OV519 chip. These are regular $\mathrm{CCD}^{6}$ USB cameras with $420 \times 640$ pixels of resolution.

The principal constraint in the design of the mobile eyes was to keep the aesthetic of the robot intact. The only visible part of the prototype is the eyes (the rest of the mechanics being hidden inside the doll's head). We use real doll eyes that we modify to insert the cameras, by drilling a tiny hole through the pupil, making sure that the iris remains intact. One DOF is assigned for coordinated sideway motion of the two eyes (to enable an eye-tracking behavior), and 2 DOFs are allocated for separate vertical motion of each eye (to enable blinking), see Figure 3.

The creation of the pair of mobile eyes was motivated by the need to identify the features of the human face which engage children with autism to interact more or less with the robot [Robins et al 2004b]. See Section 3.1 for detailed explanation of the related studies. The AURORA project has been recently provided with a Robota endowed with a prototype pair of eyes.

\footnotetext{
${ }^{6}$ Charge-Coupled Device
} 

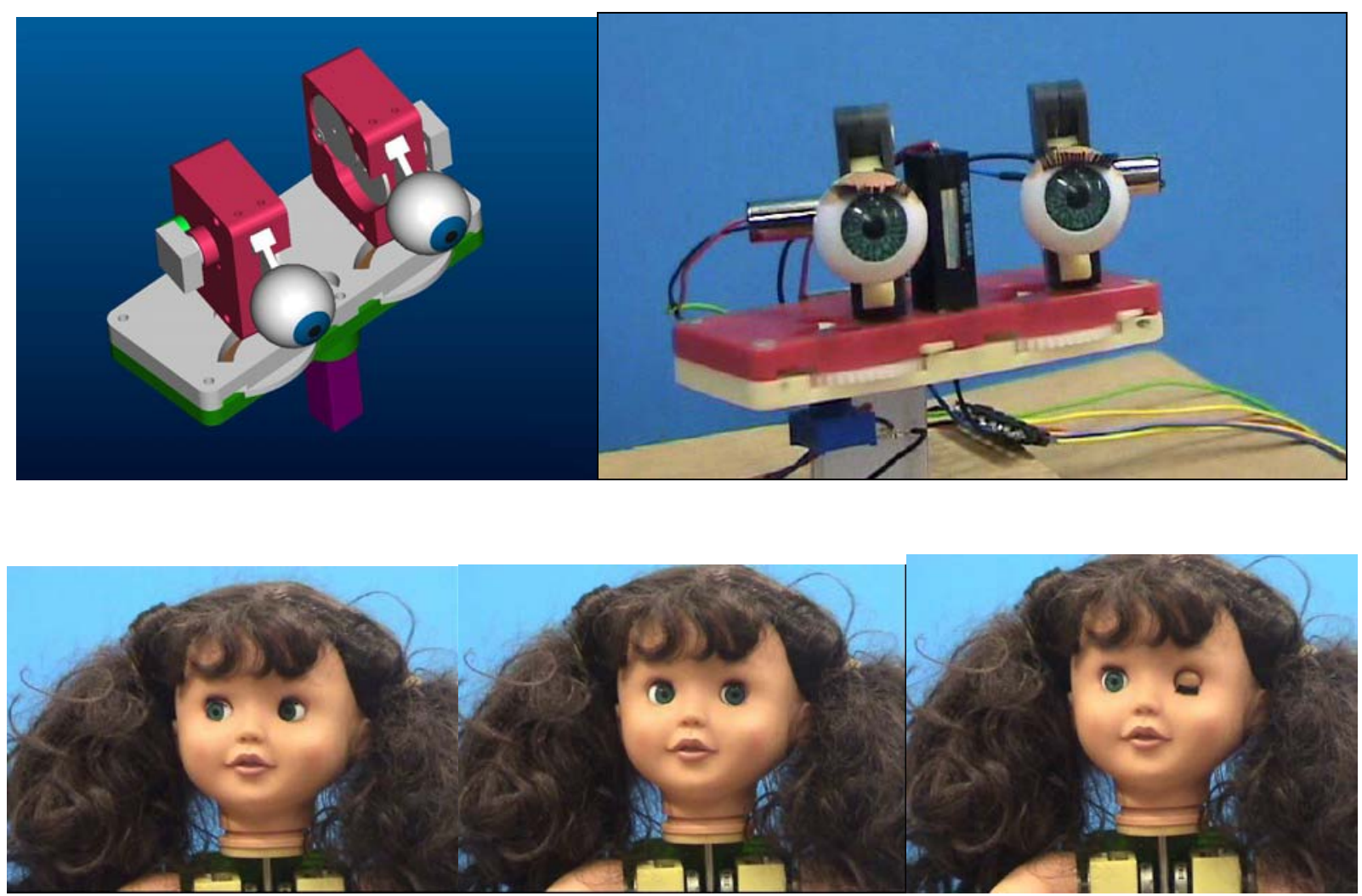

Figure 3: Prototype of 3-DOF mobile eyes for Robota.

\section{Electronics and Control}

All actuators in the eyes are simple DC Motors with digital encoders. Each motor is controlled by a Motor Module. The module allows control of position, speed and torque. The PC communicates with all the modules via I2C (Inter-Integrated Circuit). In total, we have at maximum 6 wires running through the articulation: 2 for the motors' power supply, 2 for the logic power supply and 2 for the $\mathrm{I} 2 \mathrm{C}$ communication.

\subsection{Robota's "Behaviors"}

Robota can engage in simple to complex interactions with the user, depending on whether it uses its "built-in" or "learning" behaviours. The built-in behaviours include the ability to imitate simple motions of the user's head and the arms, as well as a number of other baby-like behaviours, such as crying, being hungry/tired/happy, etc. See [Billard 2002b] for more details.

In order to engage in more complex interactions, in which the user can teach Robota, Robota is also outfitted with a number of learning behaviors. Learning is provided by an Artificial Neural Network (a time-delay associative memory), which has general properties for learning complex time series [Billard 1999]. These general learning capacities were exploited to enable the robot to engage in games in which the user can teach the robot to dance (by getting the robot to imitate its motion and learn the sequential activation of its different limbs), to learn a simple vocabulary (by associating the user's utterance with its different sensory perceptions) and to learn to "dress-up" (by associating the 
different motions required for putting on clothes with its visual perception of different clothes) ${ }^{7}$. Details on the different learning games can be found in [Billard 2002b, 2003].

In the experiments reported in Sections 3 and 4, only the imitative behaviour was being used, as described in Section 2.2. Whereas the original design aimed at providing Robota with a large variety of behaviours (assuming that it would make it more interesting and would open the way to many more interactions), early tests with Robota [Dautenhahn \& Billard 1998] showed that restricting the set of behaviours (and, thus, the set of variables to be tested) would be preferable, as it allowed the experimenters to better quantify the reactions of the children exclusively to this particular behaviour (variable). Indeed, the experimenters had more than once the occasion to verify that Autism covers a broad spectrum of disorders and that, even when taking a pre-screened subset of those children, the children's reactions to the robot vary significantly from one child to the other.

One way to compensate for this variability (especially if Robota should become a home-based therapeutic toy) may lie in exploiting Robota's learning abilities to let the robot adapt its response to the child. For instance, Robota could learn to respond (in the imitation game) more slowly to children, who might need a more guided interaction. Moreover, Robota could also adapt the game to reinforce some types of imitation (e.g. moving the head more frequently than the arms), to which the child would be more responsive.

\section{Robota's application - Educational Toy for Children with Autism:}

Robota is being used as an experimental platform for studies with children with autism by two collaborating institutions, namely the University of Hertfordshire and the University of Pierre et Marie Curie. At the University of Hertfordshire, the investigators are Kerstin Dautenhahn and Ben Robins. At the Universite Pierre et Marie Curie, the principal investigator is Jacqueline Nadel. In the following, we give an overview of the approaches and results of the work conducted by each collaborator with Robota.

For more details about each piece of work, the reader should refer to the collaborators' related publications, cited in the text.

\subsection{The Aurora Project}

At the University of Hertfordshire, Robota is used within the Aurora project (www.auroraproject.com). The Aurora project investigates the potential use of robots as therapeutic or educational 'toys' specifically for use by children with Autism. Children with autism have impaired social interaction, impaired social communication and impaired imagination [NAS 2005], referred to by

\footnotetext{
${ }^{7}$ In this instance of the game, the robot would not actually learn how to put on clothes by itself, but rather how to help the user do so, similarly to a young child. E.g, the robot would learn to lift up its arms when the user brings forth a T-Shirt and to lift up its legs when the user brings forth a pair of trousers.
} 
many authors as the triad of impairment, e.g. [Wing 1996]. Typically developing children develop the ability to appreciate the mental states of others around the third or fourth year of life. By then, most children not only have knowledge of their own mental and emotional states, but appreciate that others also have mental and emotional states of their own (theory of mind hypothesis). This ability to read others' mental states does not seem to be fully developed in children with autism and researchers suggest that children with autism have an impaired theory of mind [Baron-Cohen et al 2000, Frith 1989], and are, thus, unable to understand other people's intentions, feelings or needs.

People's social behaviour can be very complex and subtle. For a person with deficits in mental state interpretation skills, as in people with autism, this social interaction can appear widely unpredictable, and very difficult to follow. Research suggests that people with autism generally feel comfortable in predictable environments (Ferrara 1980) and enjoy interacting with various computer systems (Powell 1996; Murray 1997; Moor 1998). Interaction with robots is a multi-modal embodied interaction that can provide a simplified, safe, predictable and reliable environment, where the complexity of interaction can be controlled and gradually increased.

The Aurora project is studying if and how simple imitation and turn taking games with robots can encourage social interaction skills in children with autism, and how the robot, assuming the role of a mediator and an object of shared attention, can encourage social interaction with peers (other children with or without autism) and adults. See Figure 4
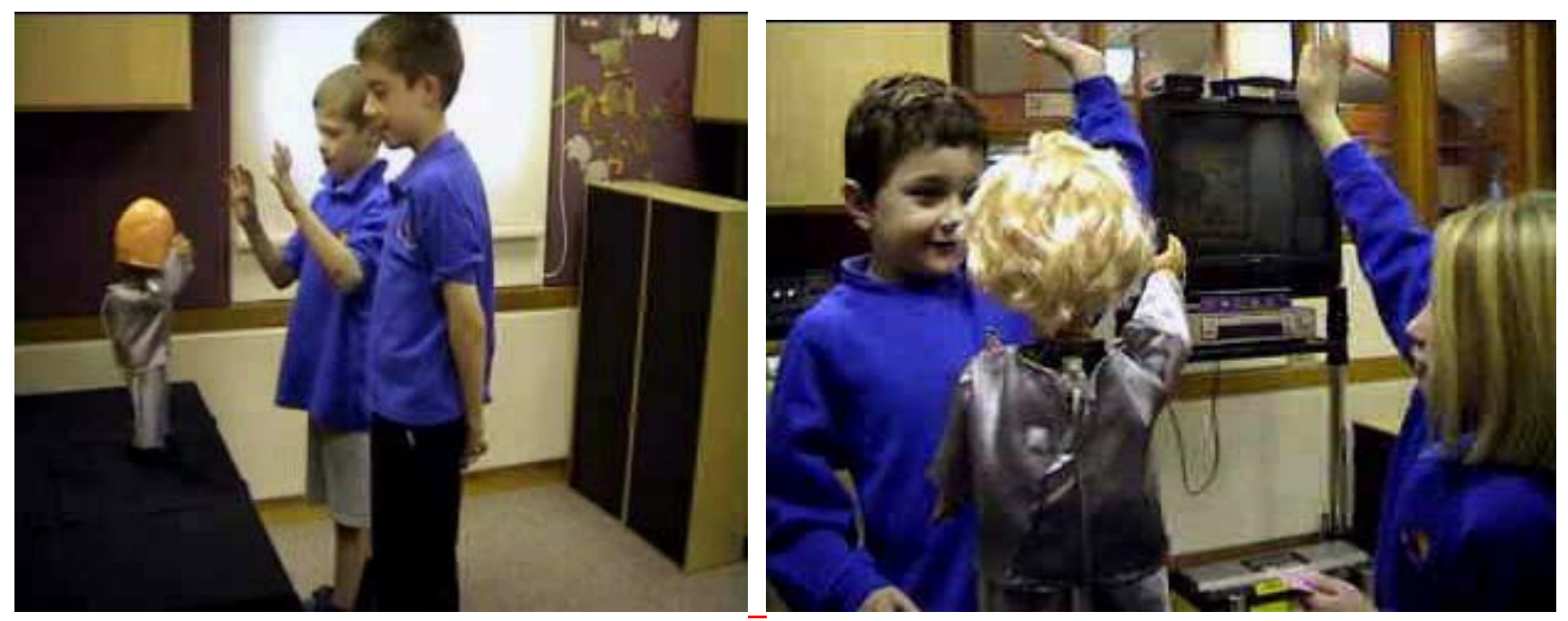

Figure 4 Robot as a mediator. The left image shows an autistic child (far end) engaged in a simple imitation game with the robot, showing his skills to another (non-autistic) child. In the right image, the robot mediates the interaction between two children with autism who are engaging in a simple imitation game with the robot.

Children participating in the various studies were aged between 5 and 7 years. They were selected by their school teachers according to the pupils' performance using the QCA's P-scale method (QCA 2003), and, more specifically, according to the pupils' personal and social development level in the subject of attention and of interaction with others. Even when scoring similarly on those categories, children would display different behaviors. One child may attend to an adult-directed activity but requires one to one support to maintain his/her attention. Another child may engage in solitary play or 
work and show little interest in the activities of those around him, whereas a third child might take part in work/play with one other person and take turns in simple activities with adult support.

The studies lasted 101 days. Few of the trials lasted up to five minutes, a few others were just under three minutes, and two ended very shortly after they started when the children left the room after 40 and 60 seconds. During this period of trials, a longitudinal repeated measure design was adopted. Unconstrained scenarios were designed, giving a high degree of freedom for the children to interact with the robot should they choose to do so. This approach also facilitated the possible emergence of spontaneous, proactive, playful interactions. In current trials, the children are freely moving around in a big room and are free to do whatever they like. The set up is inherently playful. Children with autism need a highly stable and predictable environment. Thus, the robot needs to respond with very high precision to movements of the children. Robota has therefore been used in a 'puppeteering mode', i.e. as a remote controlled robot where the experimenter picks up subtle movements of the children and responds with contingent movements of Robota's limbs and head.

\subsubsection{Effect of the Robot's Appearance:}

After initial trials with the default set up (child and teacher sitting at a table, see Dautenhahn and Billard 2002a and Figure 6), the way robota was used was changed according to the specific needs of the children with autism; see Section 2 for more details. A study into which robot design, in term of appearance, will best facilitate interactions with children with autism took place. This study contrasted the effect on the children's willingness to interact when the robot was a 'pretty girl doll' as opposed to a plain clothed doll with a featureless head, see Figure 5. The result of this study clearly indicated the children's initial preference for interaction with a plain, featureless robot over the interaction with a human, or human like robot [Robins et al., 2004b,c]. Based on these results, the robot's appearance has been changed from the default 'cute' appearance to a very plain, simpler 'robotic' appearance that is appealing to children with autism.

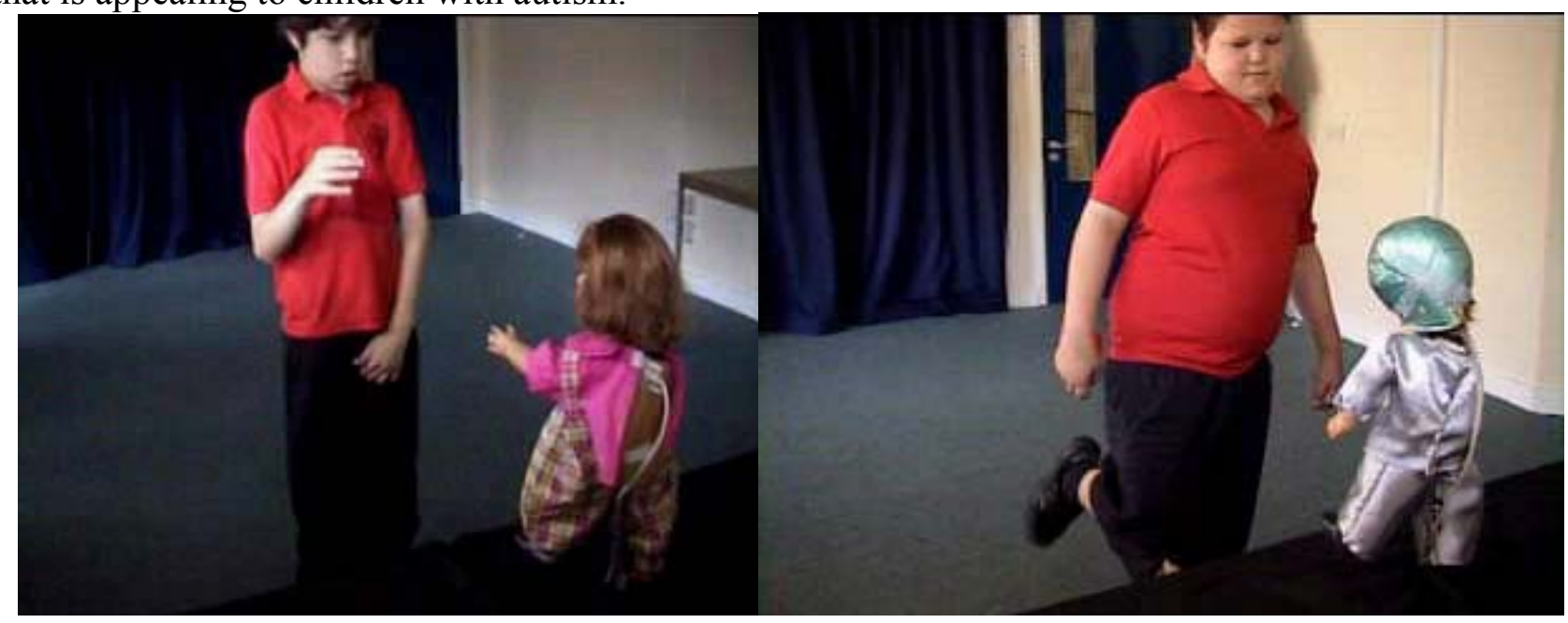

Figure 5: The robot in its two different appearances - pretty doll (left) and plain (right). Two children playing a limb-tolimb imitation game involving legs, head and arms. In this work, Robota's mirroring the motion is working in a 'puppeteering mode', i.e. as a remote controlled robot where the experimenter picks up subtle movements of the children and responds with contingent movements of Robota's limbs and head. 


\subsubsection{Qualitative Report on the Children's reaction}

Results from this longitudinal study show that the overall level of interaction with Robota (in terms of eyegaze, touch and imitation) increased over time [Robins et al 2004a]. The results also showed that Robota can elicit imitative behaviour in children with autism. Qualitative analysis of the video data, observing the children's activities in their interactional context, revealed further aspects of social interaction skills (imitation, turn-taking and role-reversal) and communicative competence that the children showed during the trials. In addition there were occurrences in these trials, where the children interacted with the robot and the investigator, using the robot as a mediator. In-depth evaluation of these occurrences showed that the children did exhibit interaction skills where the robot served as a salient object mediating joint attention with an adult [Robins et al., 2004d].

During the course of the data analysis, some issues have arisen which also showed the limitations of the approach taken in this study. These issues concern social isolation and stereotypical behaviour frequently exhibited in children with autism. They highlight the need to design interaction scenarios, and to program the robots in such a way that will ensure that the robots do encourage interaction and become a social mediator, and do not reinforce existing stereotypical behaviour, thereby becoming a social isolator [Robins et al., 2005a]. Investigating robots in the therapy and education of children with autism is a long-term research goal of the Aurora project. However, in addition to contributions to assistive technology, this work also highlights generic problems in the evaluation and design of human-robot interaction [Dautenhahn and Werry, 2002].

Autism is often referred to as autistic spectrum disorders- a range of manifestation of a disorder that can occur to different degrees and in a variety of forms. This was evident in the way the children behaved in the trials- each one of them responded differently to the robot and to the experimenter and to each other. Some liked to touch any moving part of the robot- others simply liked to observe. One child liked to sense and feel different parts of the robot against different surfaces of his body e.g he liked to rub the robot's masked head against his cheeks, or to let the robot's moving arm stroke his chest and stomach. Other children did not touch the robot at all but instead initiated imitation games with the robot, of moving arms and head, intuitively without any given prior instructions, and others did so after a demonstration of such games by their caregiver, or after given instructions by the investigator. Some children concentrated on the moving arms, and others indicated explicitly that they wanted to incorporate the legs in the game. In this stage of the research, the focus was on the fact that the robot did attract the children in the first place and interaction with it took place, and on the role of the robot in mediating interaction with other people. The research did not try to analyse in great detail the specific parts of the robot responsible for a particular response in the children.

The 'sequential process of unfolding of the interaction over time comprised three phases [Robins at al, 2004a]: a] familiarization - designed for the children to familiarize themselves with the robot if and in anyway they choose to, without any instruction from the teacher or investigator, b] learning where, if needed, the children were given instructions and/or demonstrations of simple imitation games with the robot and c] free interaction. These phases were originally designed for the longitudinal study where we investigated the effect of repeated exposure of the robot on children with autism individually. However this sequential process provde to be useful and was implemented to different degrees in all the other investigations that followed where we looked at how the robot could assume the role of social mediator in child-child interactions. Detailed qualitative analysis of 
these interactions can be found in [Robins et al, 2005b]. Here the robot provided a context for autistic children to display sophisticated embodied actions, in their response to adult requests, and in their response to the robot's movements. The robot also provided context for the children to initiate new body movements and to respond to each other's bodies using touch. The findings also highlighted the advantage of using an embodied robot rather than a computer simulation - the embodied nature of the robot allowed for the display of body orientation and full body experience in ways that a two dimensional display on a computer screen is unlikely to evoke.

\subsection{Robota at the University Pierre et Marie Curie}

Since March 2003, Robota has been included in longitudinal studies, conducted at the Hospital of LaSalpetriere and the University Pierre et Marie Curie, that investigate the use of predictive interactive machines as a means to elicit imitative behaviours in children with autism [Nadel, 2003,]. To date, these studies have revealed that children with autism, even those who are nonverbal and who have cognitive delays, are able to spontaneously imitate simple, as well as complex and novel sets of coordinated actions. Moreover, several of these children are capable of recognizing their being imitated and use this observation as a means of communicating with others [Nadel and Butterworth, 1999]. The longitudinal studies aim at exploiting these elementary skills to help a child learn the causal relationship of his/her actions on others' behaviour.

In these experiments, Robota is provided with a PocketPC and a Flycam camera. It is controlled by an algorithm for tracking and mirroring the user's hand motions [Calinon \& Billard 2003]. See Figure 6.

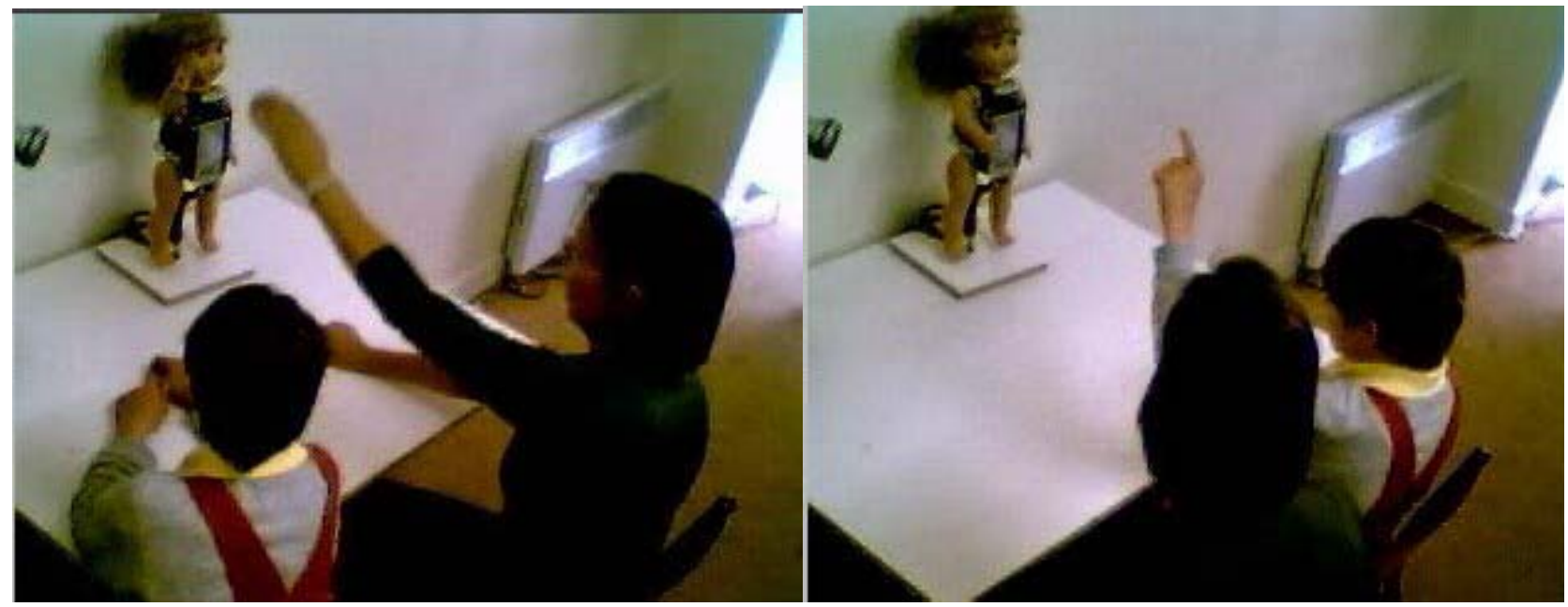

Figure 6: Left: A child with autism observes the demonstration of an imitation gesture performed by his educator. Right: The child with autism, given the freedom to explore the robot's behavior, sometimes show spontaneous imitation of the teacher's demonstration. In these experiments, Robota's mirroring of the user's arm movement is autonomous and controlled by the PocketPC and camera on-board the robot.

The game with Robota consists of two phases. In the first phase, the instructor sits in front of Robota and demonstrates the imitation game, while the child observes the instructor. In the second phase, the child sits in front of Robota. No instructions are given to the child, so that the child can demonstrate spontaneous imitation of the instructor's behaviour with Robota. 
These studies are still ongoing, but preliminary evaluation of the results have showed an increase in the interactivity and understanding of the imitation game, demonstrated through spontaneous imitation, in several children. See Figure 7. More precisely, 2 of the 10 children (age 7 to 9) tested so far immediately understood that their own movements were at the origin of Robota's motion. Five others recognized being imitated after repeated sessions and two persisted in attempts to directly move Robota's arms with their hands. These behaviours account for a different access to the concept of self-agency, as a sense of ownership of own action (Russell, 1996). Robota allows us to evaluate to what extent low-functioning children with autism are able to distinguish between perceptions being the result of their own actions and perceptions being the result of actions of others. Robota also allows the exertion of the sense of agency through repeated sessions. Moreover, "playing" with Robota encourages the children to develop an intentional selection of motor patterns in order to meet the limited repertoire of movements that Robota is capable of developing. Their recognition of being imitated by Robota is accompanied by smiles or laughs, vocalizations and communicative gestures toward Robota. 

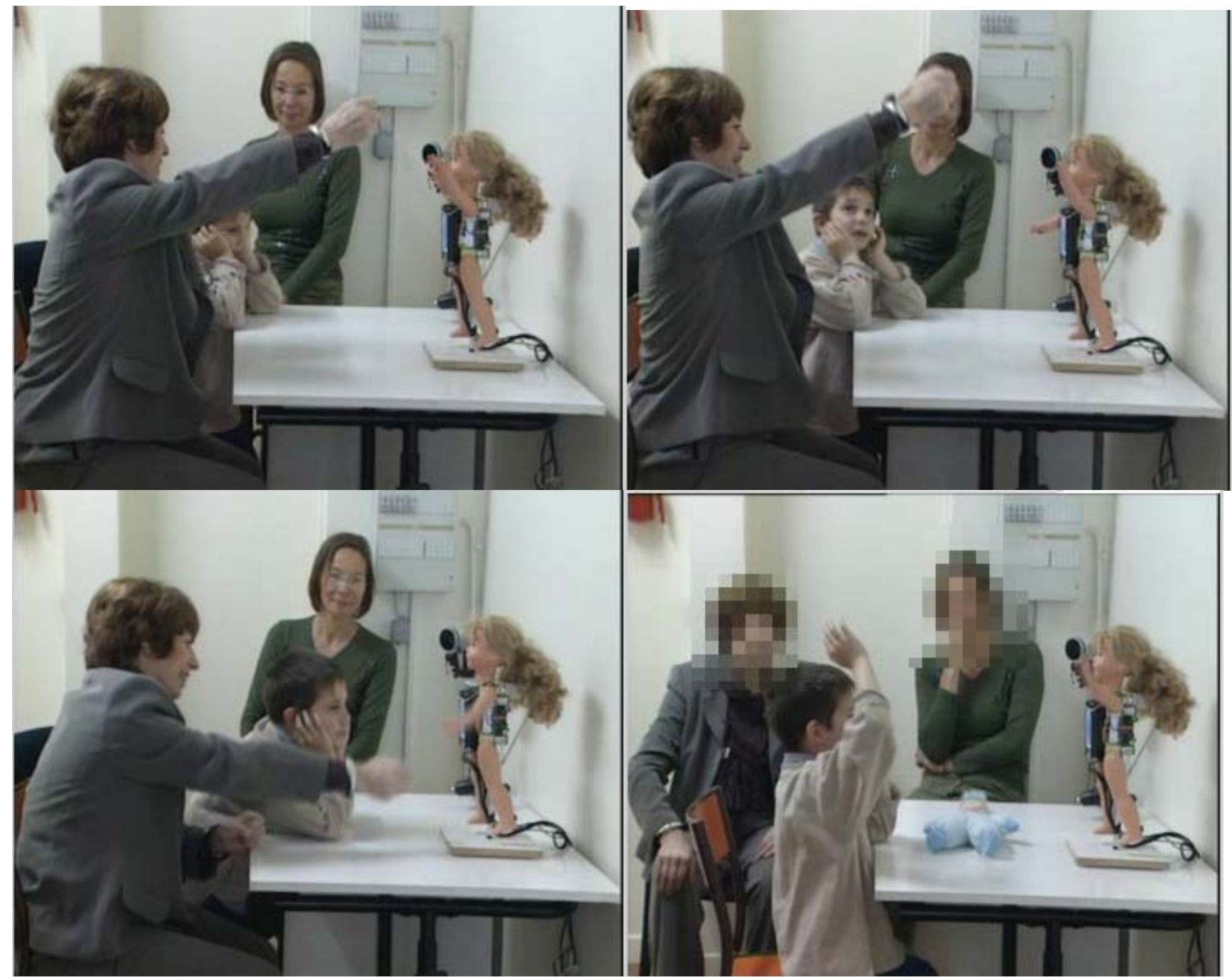

Figure 7: From top left to bottom left: Over the course of the demonstration, the child's interest in the robot grows. At the beginning of the demonstration, the child sits in a typical withdrawn attitude. At the end of the demonstration (14 seconds later), he has shifted his body forward, coming closer to the robot, looking intently at the robot. One can, also, see the child shifting his focus of attention from the robot to the demonstrator, taking in the demonstrator's arm movements and their effect on the robot's motion. Bottom Right: Once left free to play with the robot, the child spontaneously brought a doll he uses to play with on to the table to compare it to Robota, thus, demonstrating his understanding of the similarity across the two objects (the robot and the doll). He also managed in a few instances to engage in the imitation game with the robot. 


\section{Conclusion and Future Directions}

This paper has given an overview of the major goals underlying the construction and the use of the robot Robota as an assistive technology for children with autism. The Robota project is only one among several approaches, including the Aurora project, that investigate the potential of a robotics platform as part of educative therapy for children with autism. Robota complements other approaches by addressing the role that human features and imitation might have in shaping children's interaction with others. Results of such studies are promising and will set the stage for a better understanding of the needs of these children. This knowledge will inform and drive new technological development for such assistive robots.

With Robota, the reported studies led us to replace the early "invasive" infra-red set-up by a "noninvasive", vision-based mechanism for the imitation game. The vision-based interface was better tolerated by the children. Moreover, evidence suggesting that the facial features impact the children willingness to interact led us to develop a prototype with mobile eyes, that will be used, next, to test the role that eye-gaze may have on the children. Further work on Robota will aim at developing more mobile parts of the robot's head and body to test systematically which and how each of the key facial and body features affects the children's behaviour. A fundamental criterion for the design of Robota has been to maintain the overall aesthetic of the face and body intact (with respect to the original body of an attractive, child-friendly doll), in order to ensure that the effect is comparable to that of human features. This criterion will continue to be the major driving force for further development of Robota.

Since this research is still in its early stages, it is premature to derive general conclusions about the use of robotic assistive technology for autism and other similar disorders. However, the early results we reported here support the view of adopting non-invasive interfaces for interacting with the robot. Moreover, these studies suggest that one should not neglect the role that the robot's appearance (child-friendly or not) plays in engaging the child's responsiveness. Finally, early studies showed that restricting the set of behaviours of the robot to only one particular behaviour would be preferable when testing children with autism. This restriction allowed the experimenters to better quantify the reactions of the children to a particular behaviour, especially given that these children would display naturally varied and very individualized reactions toward the robot.

\section{Acknowledgements:}

Work on designing Robota was supported by the Swiss National Science Foundation; through grant no 6200066127 of the SNF Professorships program. Andre Guignard, senior technician working at the LASA Laboratory at EPFL, designed the prototype of mobile eyes for Robota. Jean-Daniel Nicoud, director of DIDEL SA, created the motor boards of Robota. The control of Robota was ported from the PC version to the PocketPC by Sylvain Calinon, PhD student at ASL3 at EPFL.

\section{References:}

Baron-Cohen, S., Tager-Flusberg, H. and Cohen, D. (2000) Understanding other minds: Perspectives 
from developmental cognitive neuroscience., Oxford University Press., Oxford.

Billard, A. (2003) Robota: Clever Toy and Educational Tool. Robotics \& Autonomous Systems, 42, 259-269.

Billard, A. (2002a) Imitation: a means to enhance learning of a synthetic proto-language in an autonomous robot. In K. Dautenhahn and C. L. Nehaniv (eds), Imitation in Animals and Artifacts, MIT Press, 281-311

Billard, A. (2002b) Play, Dreams and Imitation in Robota. In K. Dautenhahn, B. Edmonds and L. Canamero (eds), Socially Intelligent Agents, Kluwer publisher, 165-173.

Billard, A. (1999) DRAMA, a connectionist architecture for on-line learning and control of autonomous robots: Experiments on learning of a synthetic proto-language with a doll robot. Industrial Robot Journal, 26:1, 59-66, January.

Breazeal, C (2002). Designing Sociable Robots, The MIT Press.

Calinon, S \& Billard, A. (2003). PDA Interface for Humanoid Robots. Proc. of the 3rd IEEE International Conference on Humanoid Robots (Humanoids 2003), Munich and Karlsruhe.

Dautenhahn, K. and Billard, A. (2002) Games Children with Autism Can Play With Robota, a Humanoid Robotic Doll. Proc. 1st Cambridge Workshop on Universal Access and Assistive Technology (CWUAAT). In: S Keates, PJ Clarkson, PM Langdon and P Robinson (eds.) Universal Access and Assistive Technology, Springer-Verlag (London), 2002.

Dautenhahn, K, Werry, I. (2002) A Quantitative Technique for Analysing Robot-Human Interactions. Proc. IROS2002, Lausanne, IEEE/RSJ International Conference on Intelligent Robots and Systems, pp. 1132-1138, IEEE Press, 2002.

Dautenhahn, K. (1999): Robots as Social Actors: AURORA and The Case of Autism, Proc. CT99, The Third International Cognitive Technology Conference, August, San Francisco.

Frith, U. (1989) Autism: Explaining the Enigma., Blackwell. London.

Kitano, H \& Fujita, M. (2000) Robot Entertainment. In Robots for Kids: Exploring New Technologies for Learning, Druin, A \& Handler, J (eds), Morgan Kaufman Publisher.

Fujita, M, Kuroki, Y, Ishida, T and Doi, T.T. (2003) Autonomous Behavior Control Architecture of Entertainment Humanoid Robot SDR-4X. Proceedings of the 2003 IEEE/RSJ Intl. Conference on Intelligent Robots and Systems Las Vegas, Nevada · October 2003.

Ferrara C, Hill SD (1980), The responsiveness of children with autism to the predictability of social and non-social toys, Autism and Developmental Disorders 10(1):51-57. 
Guenter, F., Guignard, A., Piccardi, L., Calzascia, M. and Billard, A. (2004) Development of a miniature articulated arm and pair of eyes for the humanoid robot Robota. In Proceeding of the IEEE/APS conference on Mechatronics and Robotics.

Kozima, H., Nakagawa, C and Yano, H. (2002) Designing a Robot for Spatio-Temporal Contingency-Detection Game, International Workshop on Robotic and Virtual Agents in Autism Therapy (Hospital la Salpetriere, Paris, France), 2002.

Kozima, H (2002), Infanoid: A Babybot that Explores the Social Environment, K. Dautenhahn, A. H. Bond, L. Canamero, B. Edmonds (eds.), Socially Intelligent Agents: Creating Relationships with Computers and Robots, Amsterdam: Kluwer Academic Publishers, pp.157-164, 2002.

Kozima, H., Nakagawa, C and Yasuda, Y. (2005). Interactive Robots for Communication-Care A Case-Study in Autism Therapy, IEEE Workshop on Robots and Human Interactive Communications, Nashville, 13-15 August 2005.

Michaud, F. \& Caron, S. (2002), Roball, the rolling robot, Autonomous Robots, 12(2): 211-222.

Michaud, F., Théberge-Turmel, C. (2002), "Mobile robotic toys and autism", Socially Intelligent Agents - Creating Relationships with Computers and Robots, Kerstin Dautenhahn, Alan Bond, Lola Canamero, Bruce Edmonds (editors), Kluwer Academic Publishers, pages 125-132.

Moor D (1998) Computers and people with autism. Communication:20-21.

Montemayor, J., Druin, A. \& Hendler, J. (2000) PETS: A Personal Electronic Teller of Stories in Robots for Kids: Exploring New Technologies for Learning, Druin, A \& Handler, J (eds), Morgan Kaufman Publisher.

Murray D (1997) Autism and information technology:therapy with computers. In Powell S, Jordan R (eds): Autism and learning: a guide to good practice. London, David Fulton Publishers, p 100-117.

Nadel, J. (2005). Imitation: does it matter to children with autism? In S. Rogers \& J. Williams (Eds.), Imitation and the Development of the Social Mind. New York: Guilford Publications

Nadel, J.,Revel, A., Andry, P., \& Gaussier, P. (2004). Toward communication: first imitations in infants, low-functioning children with autism and robots. Interaction Studies: Social Behaviour and Communication in Biological and Artificial Systems, 5, 1, 45-74.

Nadel, J. (2003) Imitation et Autisme. Cerveau \& Psycho, No 4.

Nadel, J. and Butterworth, G. (1999) Imitation in infancy, Cambridge University. 
NAS (2005) National Autistic Society UK, url: http://www.nas.org.uk, last accessed 21/03/05.

QCA (2003) The qualifications and Curriculum Authority, url:

http://www.qca.org.uk/ca/foundation/profiles.asp\#p_scales Last accessed 25/11/03 Rogers

Robins B, Dautenhahn K, Dubowski J (2005a) Robots as Isolators or Mediators for Children with Autism? A Cautionary Tale. To appear in Proc. AISB05 Convention on Social Intelligent and Interaction in Animals, Robots and Agents, Hatfield, U.K, 12-15 April, 2005

Robins, B., Dickerson, P. and Dautenhahn, K.(2005b) Robots as embodied beings - Interactionally sensitive body movements in interactions among autistic children and a robot. In proc. 14th IEEE International Workshop on Robot and Human Interactive Communication -RO-MAN '05, Nashville, USA, 13-15 August 2005

Robins, B, Dautenhahn, K., te Boekhorst, R. and Billard, A. (2004a) Effects of repeated exposure to a humanoid robot on children with autism, In Keates S, Clarkson J, Langdon P, Robinson P (eds): Designing a More Inclusive World. London, Springer Verlag, p 225-236.

Robins B, Dautenhahn K, Boekhorst Rt, Billard A (2004b) Robots as Assistive Technology - Does Appearance Matter? Proc. 13th IEEE International Workshop on Robot and Human Interactive Communication - RO-MAN, Kurashiki, Japan, 20-22 September 2004.

Robins B, Dautenhahn K, Dubowski J (2004c) Investigating Children with autism's Attitudes Towards Strangers with the Theatrical Robot - A New Experimental Paradigm in HumanRobot Interaction Studies? Proc.13th IEEE International Workshop on Robot and Human Interactive Communication - RO-MAN, Kurashiki, Japan, 20-22 September 2004.

Robins B, Dickerson P, Stribling P, Dautenhahn K (2004d) Robot-mediated joint attention in children with autism: A case study in a robot-human interaction. Interaction studies: Social Behaviour and Communication in Biological and Artificial Systems, John Benjamins Publishing Company, Amsterdam 5:2:161-198.

Russell, J. (1996). Agency: its role in mental development. London: Erlbaum Taylor \& Francis

Plaisant, C., Druin, A., Lathan, C., Dakhane, K., Edwards, K., Vice, J. M., Montemayor, J.(2000). A Storytelling Robot for Pediatric Rehabilitation. ASSET'00.

Pongas, D., Guenter, F., Guignard, A. and Billard, A. (2004) Development of a Miniature Pair of Eyes With Camera for the Humanoid Robot Robota. IEEE-RAS/RSJ International Conference on Humanoid Robots.

Powell S (1996) The use of computers in teaching people with autism. In Autism on the agenda: papers. National Autistic Society Conference. London. 
Sawada, T.; Takagi, T., Fujita, M. (2004). Behavior selection and motion modulation in emotionally grounded architecture for QRIO SDR-4X II Intelligent Robots and Systems, 2004. (IROS 2004). Proceedings. 2004 IEEE/RSJ International Conference on , Volume: 3 , 28 Sept.-2 Oct., 2004 . Pages:2514 - 2519

Scassellati, B. (2005). Quantitative Metrics of Social Response for Autism Diagnosis, IEEE Workshop on Robots and Human Interactive Communications, Nashville, 13-15 August 2005.

Wing, L. (1996) The Autistic Spectrum, Constable Press, London 